Formation of Pillars at the Boundaries between HII Regions and Molecular Clouds

A. Mizuta, J. O. Kane, M. W. Pound, B. A.

Remington, D. D. Ryutov, H. Takabe

May 7, 2006

Astrophysical Journal 
This document was prepared as an account of work sponsored by an agency of the United States Government. Neither the United States Government nor the University of California nor any of their employees, makes any warranty, express or implied, or assumes any legal liability or responsibility for the accuracy, completeness, or usefulness of any information, apparatus, product, or process disclosed, or represents that its use would not infringe privately owned rights. Reference herein to any specific commercial product, process, or service by trade name, trademark, manufacturer, or otherwise, does not necessarily constitute or imply its endorsement, recommendation, or favoring by the United States Government or the University of California. The views and opinions of authors expressed herein do not necessarily state or reflect those of the United States Government or the University of California, and shall not be used for advertising or product endorsement purposes. 
revised version to ApJ, April 19, 2006

\title{
Formation of Pillars at the Boundaries between HII Regions and Molecular Clouds
}

\author{
Akira Mizuta $^{1,2}$, Jave O. Kane ${ }^{3}$, Marc W. Pound ${ }^{4}$, Bruce A. Remington ${ }^{3}$, \\ Dmitri D. Ryutov ${ }^{3}$, and Hideaki Takabe ${ }^{5}$
}

\begin{abstract}
We investigate numerically the hydrodynamic instability of an ionization front (IF) accelerating into a molecular cloud, with imposed initial perturbations of different amplitudes. When the initial amplitude is small, the imposed perturbation is completely stabilized and does not grow. When the initial perturbation amplitude is large enough, roughly the ratio of the initial amplitude to wavelength is greater than 0.02 , portions of the IF temporarily separate from the molecular cloud surface, locally decreasing the ablation pressure. This causes the appearance of a large, warm HI region and triggers nonlinear dynamics of the IF. The local difference of the ablation pressure and acceleration enhances the appearance and growth of a multimode perturbation. The stabilization usually seen at the IF in the linear regimes does not work due to the mismatch of the modes of the perturbations at the cloud surface and in density in HII region above the cloud surface. Molecular pillars are observed in the late stages of the large amplitude perturbation case. The velocity gradient in the pillars is in reasonably good agreement with that observed in the Eagle Nebula. The initial perturbation is imposed in three different ways: in density, in incident photon number flux, and in the surface shape. All cases show both stabilization for a small initial perturbation and large growth of the second harmonic by increasing amplitude of the initial perturbation above a critical value.
\end{abstract}

\footnotetext{
${ }^{1}$ Yukawa Institute for Theoretical Physics, Kyoto University, Oiwake-cho Kitashirakawa, Sakyo-ku, Kyoto, 606-8502 Japan.

${ }^{2}$ Max-Planck-Institute für Astrophysik Karl-Schwarzschild-Str. 1, 85741 Garching, Germany, Email:mizuta@MPA-Garching.MPG.DE

${ }^{3}$ University of California, Lawrence Livermore National Laboratory, 7000 East Ave., Livermore, CA 94551 U.S.A.

${ }^{4}$ Department of Astronomy, University of Maryland, College Park, MD 20742 U.S.A.

${ }^{5}$ Institute of Laser Engineering Osaka University, 2-6 Yamada Oka, Suita, Osaka, 565-0871, Japan
} 
Subject headings: HII regions - ISM: molecules - ISM: kinematics and dynamics - hydrodynamics - instabilities - methods: numerical, ISM individual object: M 16

\section{INTRODUCTION}

The shapes of the surfaces between molecular clouds and the HII regions around massive stars typically feature elongated structures, commonly referred to as pillars, columns, spikes, or elephant trunks (Hester et al. 1996; Pound 1998; Pound et al. 2003; Bally 2003). The surface is a photoionization front driven by the strong UV radiation from the OB stars. One well-known example is the Eagle Nebula which has three large, molecular pillars near a small group of O stars (Hillenbrand, Massey, Strom, \& Merrill 1993). Although a number of theoretical and numerical studies have been done, the formation mechanism is still not fully understood.

The OB stars are hot, with photospheres at temperature of a few tens of thousands of Kelvins (Dors \& Copetti 2003), and give off high intensity UV photons. These UV photons irradiate the molecular cloud which surrounds the OB stars and photoevaporation occurs, resulting in a stratified structure. The photoevaporative flow (ablated plasma) velocity is normal to the molecular surface (Hester et al. 1996). The region between the OB stars and the molecular cloud surface is the HII region in which the hydrogen gas is almost fully ionized and the photoionization and recombination to neutral atomic hydrogen occurs in steady state. The IF is a very thin layer because of the short mean free path for the incident photons on the cloud surface. At this cloud surface all the photons above the Lyman limit are absorbed. The photons below the ionization limit but above $11.2 \mathrm{eV}$ cannot ionize the hydrogen atom in the ground state but can penetrate the IF and dissociate the molecular hydrogen in the underlying layers. The thickness of the dissociation front differs with each cloud. The last layer in the stratified structure is the molecular gas. Because of strong radiative cooling, the molecular cloud temperature is typically a few tens of Kelvin (Neufeld, Lepp, \& Melnick 1995). The dynamics of such irradiated molecular clouds is thought to play a role in star formation (Bally 2003; Sugitani et al. 2002; McCaughrean \& Andersen 2002). Consequently, the outflow dynamics from the molecular cloud into the HII regions is of considerable, general interest (Pound et al. 2003; Mizuta, Yamada, \& Takabe 2002).

The pillars in M16, popularly known as the Eagle Nebula, are at a distance of $\sim 2 \mathrm{kpc}$ ((Hillenbrand, Massey, Strom, \& Merrill 1993)). It is estimated that half of the ionizing radiation comes from a single O3-4 star and the rest mostly comes from the three other nearby stars (O5-6) (Hester et al. 1996). The orientation of this system can be seen in Fig. 
1 (taken from Pound (1998)). The total ionizing flux is estimated to be $S \sim 1.2 \times 10^{50} \mathrm{~s}^{-1}$. The temperature in the HII region near the molecular cloud surface is $9500 \mathrm{~K}$ and the ionized hydrogen number density is $5 \times 10^{3} \mathrm{~cm}^{-3}$ (Levenson et al. 2000). The thickness of the photodissociation region (PDR) is relatively thin because the large hydrogen number density $\left(\mathrm{n}(\mathrm{H}) \sim 10^{3} \mathrm{~cm}^{-3}\right)$ provides high optical depth. As measured by $\mathrm{CO}$ line observations, the velocity gradients along the long axes of the pillars (from "head" to "tail") are between -20.7 and $+6.7 \mathrm{~km} \mathrm{~s}^{-1} \mathrm{pc}^{-1}$, with an average magnitude of $8.3 \mathrm{~km} \mathrm{~s}^{-1} \mathrm{pc}^{-1}$. Furthermore, the pillars are not in the same plane in the sky; the differing signs of the velocity gradients indicate inclination towards (positive gradient) or away (negative gradient) from the observer. Predictions of the classical Rayleigh-Taylor (RT) instability theory with regard to the velocity gradient (Frieman 1954) are incompatible with the observed gradient, at least for constant acceleration (Pound 1998). Another formation mechanism should be considered.

The formation mechanism of pillars beside massive stars has been discussed by a number of authors. There are two classes of models for their formation. One is that the pillars are formed due to dense, pre-existing cores in the molecular cloud (e.g. Reipurth (1983); Bertoldi \& McKee (1990); Lefloch \& Lazareff (1994); Williams, Ward-Thompson, \& Whitworth (2001)). In a interesting variation on this idea, Williams, Ward-Thompson, \& Whitworth (2001) also simulated the result of a radiatively driven, pre-existing, short pillar with the same density as the background molecular cloud (their Case II). Even in this case, the pillar grows.

Another possibility is that the pillars are caused by hydrodynamic instabilities. Spitzer (1954) proposed a model based on the RT instability occurring at the contact discontinuity between the dense molecular cloud and the lower density, hot, photoevaporated plasma. He suggested that the pillars in the Eagle Nebula were the result from the nonlinear stage of the RT instability. Frieman (1954) estimated the time scale of the instability to be less than $10^{6}$ years. Pottasch (1958) showed observations of several nebulae and estimated the age of the pillars by the RT model, assuming small initial amplitude of the perturbations. Vandervoort (1962) found another type of instability at the IF in a non-accelerating frame, the so-called IF instability, which, in his analysis, was present in the case of non-normal incident radiation. He derived a dispersion relation for perturbations growing by this process. Axford (1964) extended it to include recombination, which plays a crucial role in the HII region. The recombination in the ionized gas works to smooth the surface when the wavelength of the perturbation is much larger than the recombination length.

Williams, Ward-Thompson, \& Whitworth (2001) showed robust development of photoionized pillars with an isothermal model, although they considered a semi-infinite cloud and a non-accelerating IF. Williams (2002) derived a dispersion relation for the IF with a 
non-normal incident radiation field including the effect of recombination in the ionized gas. Ryutov et al. (2003) found unstable modes with non-normal radiation for an accelerating IF, but did not include recombination.

Most studies have assumed a semi-infinite molecular cloud and non-accelerating IF. Instabilities at an accelerating IF were studied numerically by Mizuta et al. (2005, hereafter Paper I). They show that, for imposed perturbations with small initial amplitude, i.e. in the linear regime, there was no significant growth. Large growth of a classical RT instability is observed when recombination in the HII region is not included. We study here in more depth the dynamics of an accelerating IF. The existence of the acceleration at the IF is quite different than most previous models. This paper is organized as follows. The numerical method and conditions are described in Sec. 2. The results and discussions are given is Sec. 3 , and the conclusion is given in Sec 4 .

\section{MODEL : ACCELERATING IF}

The same physics and computational method used in Paper I are included in this study. The energy balance and magnetic pressure to prevent radiative collapse for the molecular cloud are considered. The equations we numerically solve are:

$$
\begin{array}{r}
\frac{\partial \rho}{\partial t}+\nabla \cdot(\rho \boldsymbol{u})=0 \\
\frac{\partial(\rho \boldsymbol{u})}{\partial t}+\nabla \cdot(\rho \boldsymbol{u} \boldsymbol{u}+p \boldsymbol{I})=0 \\
p=\frac{2(3 f+1)}{7 f+5} \rho \epsilon+p_{M}\left(\frac{\rho}{\rho_{M}}\right)^{\gamma_{M}} \\
n \frac{\partial f}{\partial t}+n \boldsymbol{u} \cdot \nabla f=\operatorname{an}(1-f)|\boldsymbol{J}|-\alpha_{B} n^{2} f^{2} \\
\nabla \cdot \boldsymbol{J}=-a n(1-f)|\boldsymbol{J}|
\end{array}
$$

where $\rho$ is mass density, $p$ is pressure, $\boldsymbol{I}$ is unit tensor, $\boldsymbol{u}$ is the velocity vector, and $\epsilon$ is the specific internal energy. The equations (1)-(3) are mass, momentum and energy conservation with energy sources. The energy source terms due to recombination in the ionized region, absorption of the UV radiation from OB stars, and cooling in the molecular gas are $q_{r e}, q_{u v}, q_{m o l}$, respectively. These energy sources are evaluated as

$$
q_{r e}=(n f)^{2} \beta_{B} k T,
$$




$$
\begin{aligned}
& q_{u v}=\operatorname{Wan}(1-f)|\boldsymbol{J}|, \\
& q_{m o l}=n_{m o l}^{2} \times 10^{-29} \mathrm{erg} \mathrm{cm}^{-3} \mathrm{~s}^{-1} \text {, } \\
& T=\left(m_{p} / k\right) \times[4 \epsilon /(7 f+5)], \\
& n_{m o l}=n(1-f) / 2 \text {, } \\
& \rho \epsilon=3(n f) k T+2.5 n_{m o l} k T,
\end{aligned}
$$

where $T$ is the temperature in Kelvins $m_{p}$ is the proton mass, $k$ is the Boltzmann constant, and $n$ and $n_{m o l}$ are atomic hydrogen and molecular hydrogen number density. We ignore metal line cooling for simplicity, since its cooling power has the same dependence as that of recombination (proportional to ionized hydrogen number density). The dissociation heating in the neutral region and other radiative processes are also ignored for simplicity. The heating function is $W=1.73 \times 10^{-12} \mathrm{erg}$ which corresponds to the average energy deposited into the gas per absorbed ionizing photon, and leads to produce an isothermal temperature of $T=10^{4}$ $\mathrm{K}$ in the ionized gas as an equilibrium state of photoionization heating and recombination cooling. The case B recombination coefficients, which are summation of all recombination coefficients of hydrogen except the recombination to the ground state, are assumed constant $\alpha_{B}=2.6 \times 10^{-13} \mathrm{~cm}^{3} \mathrm{~s}^{-1}$ and $\beta_{B}=1.25 \alpha_{B}$ (at $T=10^{4} \mathrm{~K}$ from Hummer \& Seaton (1963)), where $\beta_{B}$ includes the thermal velocity dependence of the rates of recombination and freefree collisional cooling. The equation of state (Eq. 4) includes a magnetic pressure term for dense gas to prevent radiative collapse (Ryutov \& Remington 2002 ), where $f \equiv n_{i} / n$ is the ionization fraction, $n_{i}$ is the ionized hydrogen volume density, $p_{M}$ and $\rho_{M}$ are constant values. The index $\gamma_{M}(=4 / 3)$ is also constant. Numerically, we do not take into account the atomic hydrogen state, assuming the dissociation front is thin as in the Eagle nebula. Equations (5) and (6) describe the evolution of $f$ and the transport of the incident radiation, where $a=6 \times 10^{-18} \mathrm{~cm}^{2}$ is the photoionization cross-section of hydrogen, and $\boldsymbol{J}$ is the number flux of ionizing photons, i.e., photons $\mathrm{cm}^{-2} \mathrm{~s}^{-1}$. Since we use the "on-the-spot approximation" (i.e., photons emitted in recombination to ground state are immediately reabsorbed, whereas the photons emitted in recombination to second or higher levels are assumed to escape from the system), we do not consider the diffusive photon emission and transport.

The $2 \mathrm{D}$ computational domain is $(x \times y)=(0.46 \mathrm{pc} \times 3 \mathrm{pc})$. Uniform grid points $(\Delta x=$ $\left.\Delta y=2.5 \times 10^{-3} \mathrm{pc}\right)$ are used. Periodic boundary conditions are employed $(x=0$ and $x=0.46 \mathrm{pc}$ ). The other boundary conditions are open boundary which means zero gradient. An incident photon flux $\left(\boldsymbol{J}_{\mathbf{0}}=-5 \times 10^{11} \hat{y} \mathrm{~cm}^{-2} \mathrm{~s}^{-1}\right)$ is imposed from one boundary $(y=3$ pc), where $\hat{y}$ is the unit vector of the $y$ axis. The rays are parallel to the $y$ axis. A cloud of initial thickness of a quarter pc, and density of $n\left(H_{2}\right)=10^{5} \mathrm{~cm}^{-3}$ is set $0.5 \mathrm{pc}$ away from the boundary where the incident photons come in. A very dilute gas $n(H)=10 \mathrm{~cm}^{-3}$ is imposed in the other regions. Initially, the gas at $y>2.25 \mathrm{pc}$ is in pressure equilibrium, 
and the gas at $y<2.5 \mathrm{pc}$ is isothermal $(40 \mathrm{~K})$. The constant parameters in the equation of state, such as, $\rho_{M}$ and $p_{M}$, are the mass density and thermal pressure, of the initial molecular cloud, respectively.

We impose the perturbation in the cloud in three different ways. The first is a density perturbation in a layer of $0.125 \mathrm{pc}$ thickness along the surface of the cloud, with the form $n=n_{0}(1-A \cos [2 \pi x / 0.46 \mathrm{pc}])$, where $A$ is the amplitude of the perturbation and $n_{0}=$ $10^{5} \mathrm{~cm}^{-3}$. We assume a wavelength of $\lambda=0.46 \mathrm{pc}$, and consider amplitudes $A=0.2$ (model D2), 0.3 (D3), 0.4 (D4) and 0.5 (D5). The second method is a $30 \%$ amplitude perturbation in the incident photon number flux of form $\boldsymbol{J}=\boldsymbol{J}_{\mathbf{0}}(1-0.3 \cos [2 \pi x / 0.46 \mathrm{pc}])$, starting at 98, 102, 106, or $108 \mathrm{kyr}$ and ending at $110 \mathrm{kyr}$. These are models P098, P102, P106, P108, respectively. This is similar to the method used in Paper I and in Mizuta et al. (2005b). The third method is to impose surface perturbations on the initial cloud, according to $y=2.5-C \cos [2 \pi x / 0.46 \mathrm{pc}]$. The amplitude $(C)$ is $3.8 \times 10^{-3} \mathrm{pc}$ (model S038), $9.0 \times 10^{-3}$ pc $(\mathrm{S} 090), 1.4 \times 10^{-2}$ pc $(\mathrm{S} 140)$, and $1.9 \times 10^{-2}$ pc $(\mathrm{S} 190)$.

\section{RESULTS and DISCUSSION}

\subsection{D Dynamics}

We briefly show the results without any perturbations, before showing the results with perturbations. The dynamics without any perturbation is very simple. When the incident photon flux is turned on, a shock propagates through the molecular cloud (compression phase). After this shock breaks out of the back side of the cloud, a rarefaction passes back through the shocked cloud. Then an acceleration phase begins at about $t=100 \mathrm{kyr}$, as the cloud moves as a unit. Figure 2 shows one dimensional profiles of the hydrogen number density at the early phase of the dynamics at $t=0,50,100,110$, and $150 \mathrm{kyr}$. In the figure, all three phases are shown, although the phase at around $t=100 \mathrm{kyr}$ to $t=110 \mathrm{kyr}$ in which the rarefaction passes back through the compressed cloud is too short to show.

The hydrogen number density profiles of an initially semi-infinite cloud are shown in Fig. 3, both with and without magnetic pressure. The initial total pressure without the magnetic component is lower than with it. Without magnetic pressure, the number density of the compressed cloud becomes about 1.5 times higher, since there is less pressure support. 


\subsection{Small Initial Amplitude Cases}

Figure 4 shows the evolution of the amplitude, i.e., half the peak-to-valley of the perturbation of the $f=0.5$ contour in the $y$ direction for each case, for all three methods of imposing the perturbation. It should be noted that this does not always correspond to the amplitude of the perturbation on the cloud surface because of the effect of "separation of the IF" discussed below. In cases with small initial perturbations (models D2, D3, P106, P108, and S038), the perturbation does not grow but rather oscillates as observed in Paper I. This stabilization is caused by the density profile in the HII region. The ablated (photoevaporated) gas in the concave region of the incipient bubble concentrates (gets weakly "focused") in the region above the bubble vertex. This concentration of gas subsequently absorbs more of the incoming ionizing photons, reducing the photon flux reaching the IF region at the cloud surface near the bubble vertex, compared with the spike region. This is because the recombination rate depends on the square of the ionized hydrogen number density in the HII region. The net result is that the ablation pressure locally decreases in the bubble region compared to the spike region, which acts to smooth out the perturbed surface, if the perturbation is small. This is also shown in Fig.5a with a 2D plot of number density late in time $(t=460 \mathrm{kyr})$ for a small initial density perturbation (model D2). The IF is quite smooth, namely, no growth of the perturbation has occurred. The other cases (D3, P106, P108, and S038) in which the perturbation does not grow behave similarly.

\subsection{Large Initial Amplitude Cases}

When the perturbation initial amplitude is larger, roughly when the ratio of the initial amplitude to wavelength is greater than 0.02, the perturbations grow, after the acceleration phase begins at around $t=100$ kyr (models D4, D5, P098, P102, S090, S140, and S190). Recombination in the HII region is included in all cases. Here, we concentrate on the case with an imposed density perturbation (model D5) to illustrate why the perturbation grows when the amplitude of the imposed perturbation exceeds a critical value.

Figures 5 (b)-(i) show a series of the 2D hydrogen number density plots (color) with incident photon flux contours (solid curves) at different times. The high density regions correspond to molecular gas compressed by the ablation pressure. A typical hydrogen number density in the HII region near the IF is $10^{3} \mathrm{~cm}^{-3}$ as shown in Figure 2. The solid white lines correspond to incident photon number contours from 0 to $5 \times 10^{11} \mathrm{~cm}^{-2} \mathrm{~s}^{-1}$ at intervals of $1 \times 10^{11} \mathrm{~cm}^{-2} \mathrm{~s}^{-1}$, starting from the ionization front where the ionization fraction $(f)$ goes to zero. Since we do not show the whole computational domain, all the photon flux contours are not shown in the figures in the later phases. 
In Fig.5 (b), the photon flux contours near to the IF are of the opposite phase as the IF because of the oscillation of the IF. This oscillation occurs by the stabilization mechanism seen in the linear regime (Paper I). In Fig.5 (c), the $|\boldsymbol{J}|=0$ photon flux contour separates slightly from the highly compressed molecular cloud around $x=0.23 \mathrm{pc}, y=2.3 \mathrm{pc}$ (see also the close-up view in figure 6). This is the separation of the IF from the cloud surface. It happens because all the incident photons moving toward this region are absorbed by the recombined neutral hydrogen accumulated near the bubble vertex at $x=0.23 \mathrm{pc}$. This could be understood as an extreme case of the normal stabilization mechanism of the IF instability, because the increasing density around the cavity causes strong absorption of the incident photons. During the separation of the IF, the cloud surface is shadowed. This shadowed region does not feel any ablation pressure because no absorption of the incident photons occur there, whereas the other parts of the cloud surface which are directly ablated by the incident photons feels strong ablation pressure.

The region between the shadowed cloud surface and the separated IF becomes neutral. The appearance of the neutral region happens because the timescale for recombination is roughly $\left(\alpha_{B} n_{i}\right)^{-1} \sim 10^{2}$ yr for ionized hydrogen at a number density of $n_{i}=10^{3} \mathrm{~cm}^{-3}$. Thus, once the cloud surface is shadowed, the neutral region quickly appears close to the shadowed surface. We can identify this region as a warm HI region $(T \sim 4000 \mathrm{~K})$ because it has lost half of its thermal energy by recombination cooling. The pressure in this warm HI region is a few times smaller than that of the HII region and the molecular gas. This region has similar physical properties as the warm HI layer in front of PDRs described by Hollenbach \& Tielens (1997) (see their Figure 3), which also arises due to a lowering of the ionization fraction.

The appearance of this separation strongly affects the dynamics of the IF. In reaction, the compressed cloud expands into the low pressure region, namely, in the $y$ direction, which triggers the second harmonic of the imposed initial perturbation in subsequent frames (Fig.5(d) - (e)). Although the second harmonic of the imposed initial perturbation of the cloud surface can be clearly seen at $t=200 \mathrm{kyr}$, the second photon contour $(|\boldsymbol{J}|=1 \times$ $10^{11} \mathrm{~cm}^{-2} \mathrm{~s}^{-1}$ ) from the IF still shows just a single mode. In other words, the density around the side $(x=0,0.46 \mathrm{pc})$ is high, and the density around the center $(x=0.23)$ is low, although both regions on the cloud surface are spikes. Thus the stabilization mechanism seen in the linear regime (Paper I, and section 3.2) does not work in this case. The ablation pressure is strong around the center $(x=0.23 \mathrm{pc})$, because the two last contours are close to each other there (meaning the photon flux incident on the cloud surface is relatively high). As a result, a large amount of thermal energy is deposited there and compression by the strong ablation pressure occurs. By contrast, around the side $(x=0,0.46 \mathrm{pc})$ the last two incident photon contours are separated from each other (meaning a lower photon flux reaches 
the ablation front at the cloud surface). The resulting lower ablation pressure causes slight local expansion in the $y$ direction (Fig.5 (e) and (f)). This expansion allows the growth of the spike at the sides (Fig.5 (g)), and a strong second harmonic has grown up.

By $320 \mathrm{kyr}$, separation of the IF from the ablation front (cloud surface) near the lateral boundaries has occurred (Fig.5(h)), and by 400 kyr (Fig.5(i)), this separation has also appeared near the middle. The net result is that the perturbation amplitudes at the center and lateral edges grow. Once a critical amplitude is exceeded, at around $400 \mathrm{kyr}$, the stabilization mechanism is weakened by the steepness of the perturbations (i.e., the photon flux and therefore ablation rate along the steep sides of the perturbations drops, so there is much less density rise in the HII gas in the concave region), and the RT evolution proceeds rapidly into the deep nonlinear regime (Fig.5(j)).

For the other cases with a large amplitude of initial perturbations (D4, P098, P102, S090, S140, and S190), we observe similar dynamics of the IF and cloud surface. The separation of the IF occurs, a second harmonic of the imposed perturbation is triggered, and finally two pillars grow. The center panels of Fig. 7 show a large perturbation growth in later phase of the dynamics using the different methods of the imposing the initial perturbation : density modulation (model D5; top), perturbation in photon number flux (model P102; middle), and surface perturbation (model S090; bottom). The growth of the second harmonic mode of the perturbation is observed in all cases.

\subsection{Velocity Gradient}

Figure 8 shows the $y$-component of the velocity in the pillar for an initial perturbation in density (model D5), in photon flux contour (P102), and in the surface (S090). The $V_{y}$ gradients are $\sim 8.0(\mathrm{D} 5), 16(\mathrm{P} 102)$, and $12(\mathrm{~S} 090) \mathrm{km} \mathrm{s}^{-1} \mathrm{pc}^{-1}$. In a real pillar, an observer would see any velocity gradient corresponding to $V_{y} \sin (i)$, where $i$ is the inclination angle of the pillar with the plane of the sky. Indeed, Pound (1998) measured such gradients in the pillars of the Eagle Nebula. This observed velocities in Pillar II of the Eagle Nebula are also plotted for comparison. For the purposes of the figure, we have taken $i=15$ degrees, dividing the observed values by $\sin (15)$. Thus, for small inclination angles, our numerical results are good agreement with the observed Pillar II velocity gradient of $2.2 \mathrm{~km} \mathrm{~s}^{-1} \mathrm{pc}^{-1}$ (Pound (1998)). 


\subsection{Resolution Effects}

The calculation without the recombination effect, which was shown in Paper I, shows a large growth of the imposed perturbation. The surface also shows growth of a shorter wavelength perturbation due to purely numerical reasons, such as less dissipation. It is impossible to impose exactly one single mode using Cartesian coordinates; small amplitude and short wavelength perturbations will also always be imposed. The wavelength of the shortwavelength "noise" which grows depends on the resolution of the calculation. To eliminate the possibility of this effect in the current calculations, we also performed the calculations with higher and lower resolution in the $x$ direction; specifically, twice (384) and a half (96) the number grid points than in our standard calculation. Figure 7 shows the results of higher (right panels) and lower (left panels) resolution calculations. As a reference, the results of the standard resolution calculation are also shown (center). The results of the three different ways for imposing perturbations are shown (top : $t=460 \mathrm{kyr}, 50 \%$ peturbation in density; middle : $t=440 \mathrm{kyr}, 30 \%$ peturbation in photon number flux during 102-110 kyr; bottom : $t=460 \mathrm{kyr}$, surface perturbation of amplitude $9 \times 10^{-3} \mathrm{pc}$ ). The pillars appear and grow at all resolutions, differing only in fine-scale structure at the cloud surfaces. The separation of the ionization front is also observed at the early phase of the dynamics in each case. Thus, we conclude that the pillar appearance is not a resolution-dependent effect.

\subsection{Discussion}

That we produce pillars is purely the result of our periodic boundary conditions. Unlike the pillars in our simulation, the Eagle pillars are not co-planar and do not share the same inclination angle (Pound (1998)). They are effectively three isolated pillars, rather than a single structure. Pillars in other HII regions are also typically isolated. However, our main aim in this paper has been to describe the instability of accelerating IF, so we have assumed initially a single wavelength and periodic boundary conditions. We have chosen only one set of free parameters - the wavelength and number density, initial magnetic pressure of the initial cloud, incident photon number flux at the boundary, and thickness of the cloudalthough these specific values match those of Eagle Nebula reasonably well.

The main key to this instability is the mismatch of perturbation modes between the IF and density in the HII region. The separation of the IF from the cloud is the trigger for

this mismatch. Thus, it should still be possible to create an isolated pillar in the HII region under conditions where a single wavelength grows and becomes a pillar. Different wavelengths and multiwavelength cases should be investigated, however, since the phenomenon relies on nonlinear dynamics. 
The effect of varying the magnetic field pressure should also be investigated, although the magnetic pressure is introduced just to prevent too high a number density from forming and does not affect the physical mechanism of the growth of the perturbation. An MHD calculation is one avenue for improvement over the current models.

In this study we have adopted the on-the-spot approximation which ignores the recombination of the ionized hydrogen to the ground state, assuming that the photon emitted by this recombination is absorbed locally because its energy is high enough to ionize another hydrogen atom. However, this approximation is not sufficient for Cartesian calculations when the directions of IF propagation and of the incident light rays become close to parallel. In such cases, irradiation by diffuse photons is the main source of ionization of the sides of the pillars. We expect to include this diffused photon transport in future calculations.

\section{CONCLUSION}

We have investigated hydrodynamic instability of the accelerating IF including detailed energy accounting. Three different ways of imposing the initial perturbation were adopted: (1) perturbation in density: (2) perturbation in photon number flux: and (3) surface perturbation.

We observe no large growth when the incident perturbation is small. This stabilization is the same as that observed in Paper I, and identical to the stabilization mechanism theoretically found by Axford (1964) for the non-accelerating IF. The strong absorption of incident photons causes decreasing ablation pressure around the cavity, which effectively smooths out small amplitude perturbations.

In contrast, large growth of the second harmonic of the imposed perturbation is observed when the initial perturbation is large, namely, the ratio of the initial amplitude to wavelength is greater than 0.02. A slight separation of the IF from the cloud surface triggers the appearance of a warm HI region and the appearance of a second harmonic of the imposed perturbation. The separation of the IF from the cloud surface happens when all the incident photons traveling toward this region are absorbed by the recombined neutral hydrogen accumulated near the bubble vertex. The gas at this cloud surface at location begins to expand in the $y$ direction during the separation because the pressure in the warm HI region is less than that in other regions. Although the second harmonic is imprinted on the cloud surface, the density perturbation and incident photon flux contours in the HII region are still single mode. The mismatch between modes at the cloud surface and in the density in the HII region above the cloud surface prevents stabilization and cause nonlinear 
dynamics of the molecular cloud. In the later phase of the dynamics, large growth of the perturbation is observed.

This new result for the IF instability does not depend on the method by which the initial perturbation is imposed. We also show that it is independent of the resolution of the calculation. The velocity gradient in the model pillar in the later phase of the dynamics is in good agreement with that observed in the Eagle Nebula.

We would like to thank to Takashi Hosokawa, Mark Wolfire, Nobuhiko Izumi, and Robin Williams for useful discussions and comments. We acknowledge the anonymous referee for helpful comments which improved this manuscript. This work was performed under the auspices of the U.S. Department of Energy by the Lawrence Livermore National Laboratory under Contract No. W-7405-ENG-48 and with support from NASA Grant NRA 00-01-ATP059 and from National Science Foundation under Grant No. AST-0228974.

\section{REFERENCES}

Axford, W. I. 1964, ApJ, 140, 112

Bally J. \& Reipurth B. 2003, AJ, 126, 893

Bertoldi, F., \& McKee, C. F. 1990, ApJ, 354, 529

Dors, O. L., \& Copetti, M. V. F. 2003, A\&A, 404, 969

Frieman, E. A. 1954, ApJ, 120, 18

Hester, J. et al. 1996, AJ, 111, 2349

Hillenbrand, L. A., Massey, P., Strom, S. E., \& Merrill, K. M. 1993, AJ, 106, 1906

Hollenbach, D. J., \& Tielens, A. G. G. M. 1997, ARA\&A, 35, 179

Hummer, D. G. \& Seaton, M. J. 1963, MNRAS, 125, 437

Lefloch, B., \& Lazareff, B. 1994, A\&A, 289, 559

Levenson, N. A., et al. 2000, ApJ, 533, L53

McCaughrean, M. J., \& Andersen, M. 2002, A\&A, 389, 513

Mizuta, A., Yamada, S., \& Takabe, H. 2002, ApJ, 567, 635 
Mizuta, A., et al. 2005a, ApJ, 621, 803 (Paper I)

Mizuta, A., et al. 2005b, Astrophysics and Space Science, 298, 197

Neufeld, D. A., Lepp, S., \& Melnick, G. J. 1995, ApJS, 100, 132

Pottasch, S. R. 1958, Reviews of Modern Physics, 30, 1053

Pound, M. W. 1998, ApJ, 493, L113

Pound, M. W., Reipurth, B., \& Bally, J. 2003, AJ, 125, 2108

Reipurth, B. 1983, A\&A, 117, 183

Ryutov, D. D. \& Remington, B. A. 2002, Plasma Physics and Controlled Fusion, 44, 407

Ryutov, D. D., Kane, J. O., Pound, M. W., \& Remington, B. A. 2003, Plasma Physics and Controlled Fusion, 45, 769

Spitzer, L. J. 1954, ApJ, 120, 1

Sugitani, K., et al. 2002, ApJ, 565, L25

Vandervoort, P. O. 1962, ApJ, 135, 212

Williams, R. J. R., Ward-Thompson, D., \& Whitworth, A. P. 2001, MNRAS, 327, 788

Williams, R. J. R. 2002, MNRAS, 331, 693

This work was performed under the auspices of the U.S. Department of Energy by University of California, Lawrence Livermore National Laboratory under Contract W-7405-Eng-48. 


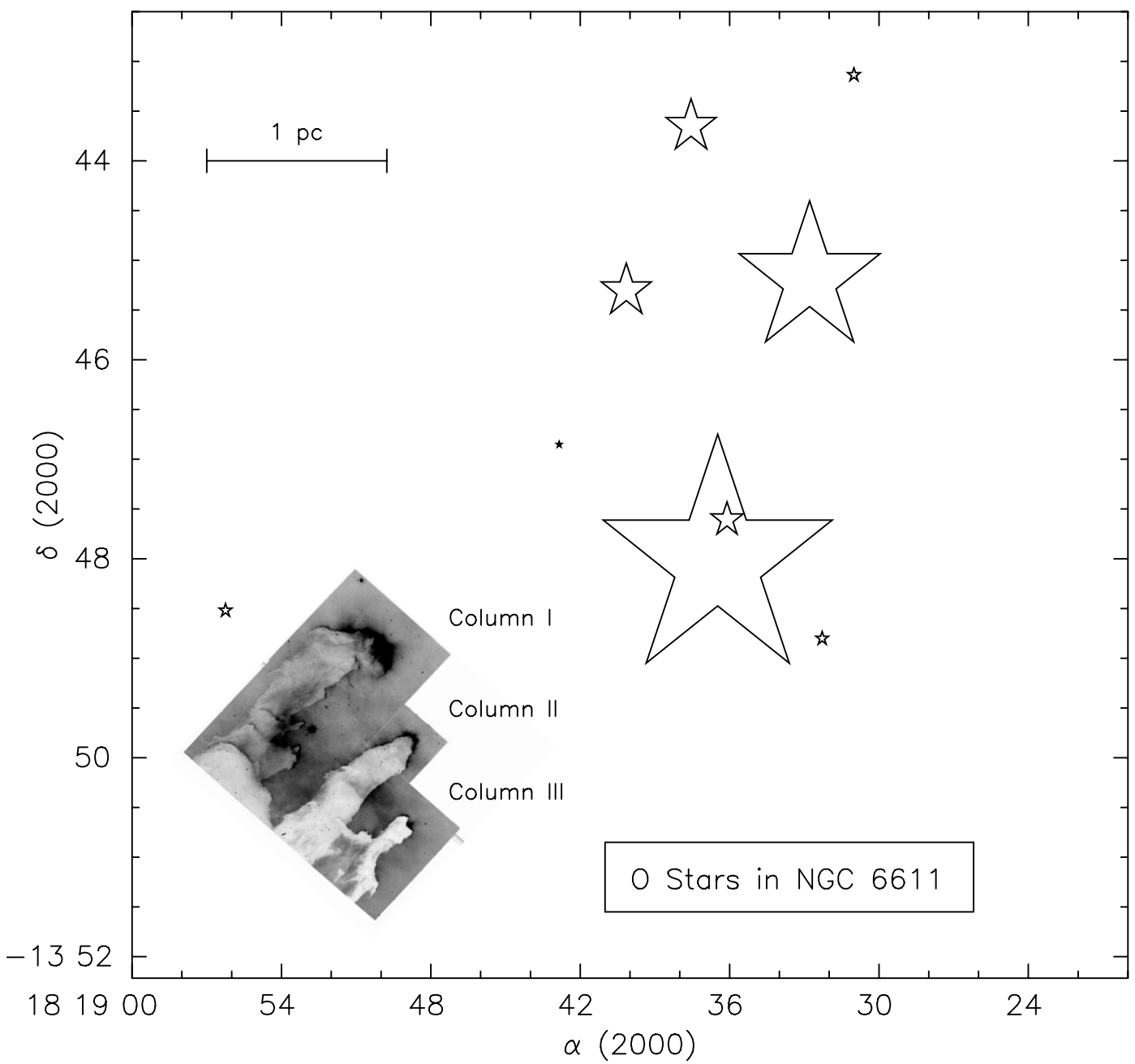

Fig. 1.- Schematic diagram showing the position of the O stars that are photoevaporating the Eagle Nebula molecular cloud taken from Fig. 1 in Pound (1998). The size of the symbols indicates the relative strength of the Lyman continuum flux from each star. Most of the ionizing radiation comes from two stars of spectral type $\mathrm{O} 5 \mathrm{~V}$ and $\mathrm{O} 5.5 \mathrm{~V}$ in the nearby young cluster NGC 6611. 


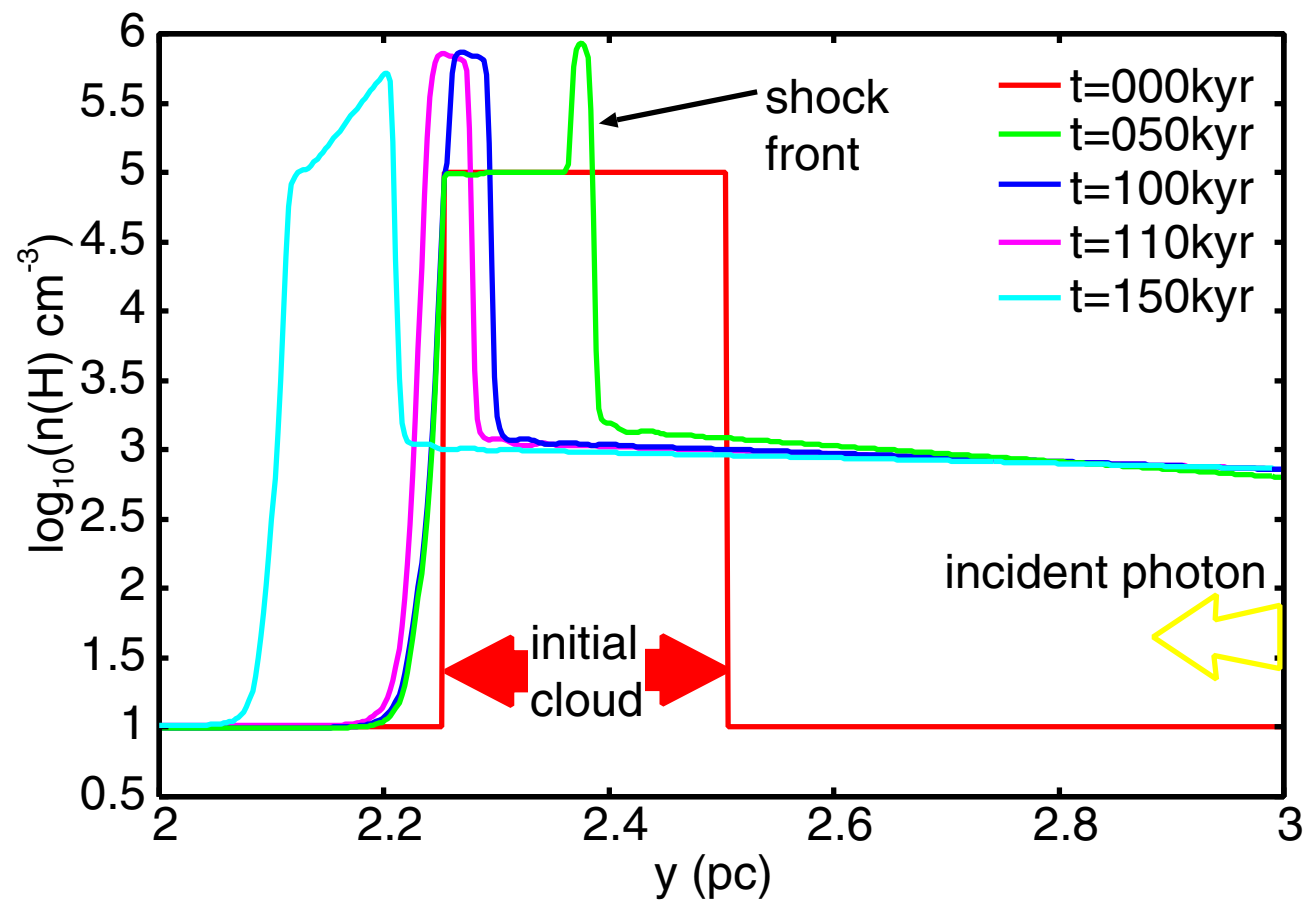

Fig. 2.- One dimensional hydrogen number density profiles of the case without perturbations at five different times, $t=0,50,100,110$, and $150 \mathrm{kyr}$. The profiles at three phases of the dynamics, compression $(0 \leq t \lesssim 100 \mathrm{kyr})$, rarefaction $(100 \lesssim t \lesssim 110 \mathrm{kyr})$, and acceleration $(t \gtrsim 110 \mathrm{kyr})$ are shown. 


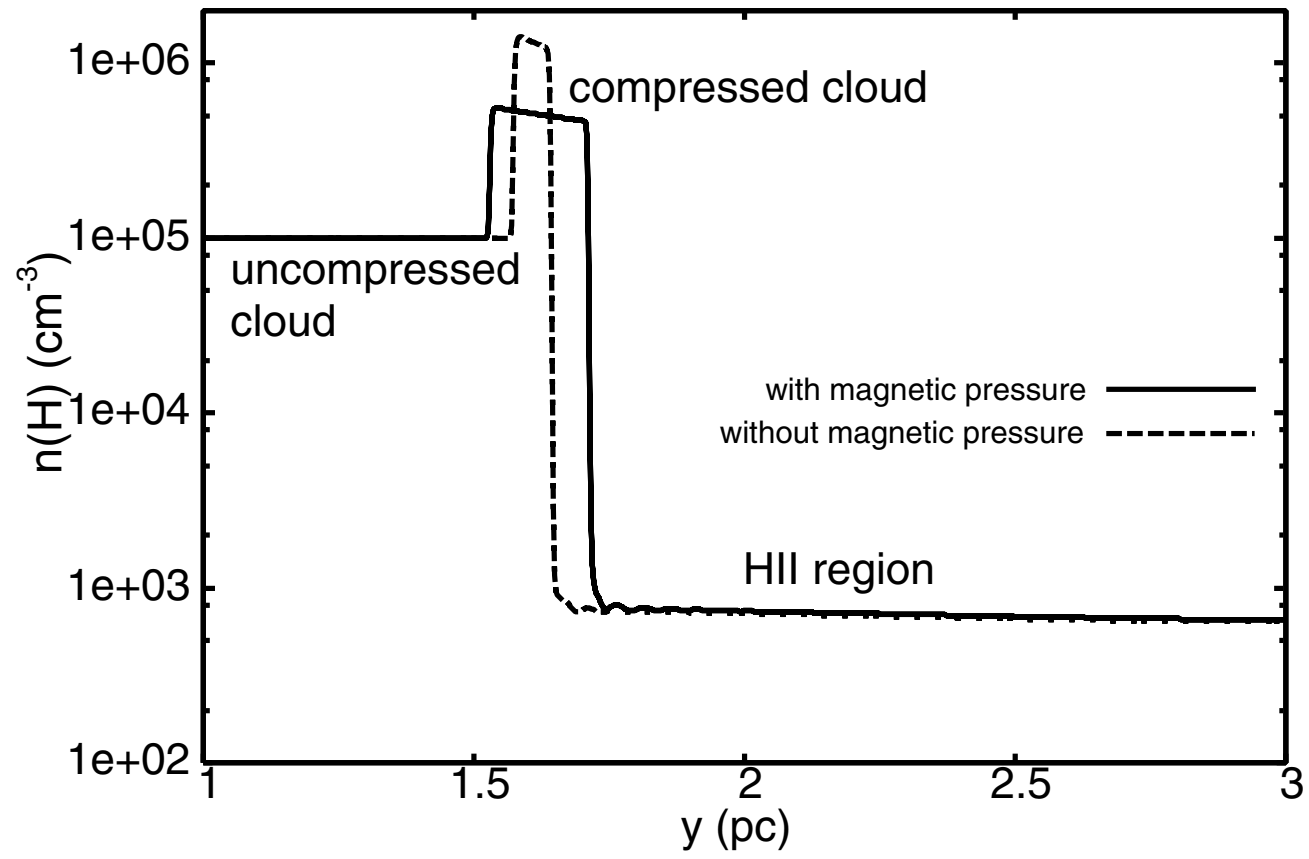

Fig. 3.- One dimensional hydrogen number density profiles of the initial semi-infinite cloud. The cases with and without magnetic pressure at $t=500 \mathrm{kyr}$ are shown. The number density of the compressed cloud without magnetic pressure is about 1.5 times higher than that with magnetic pressure. 


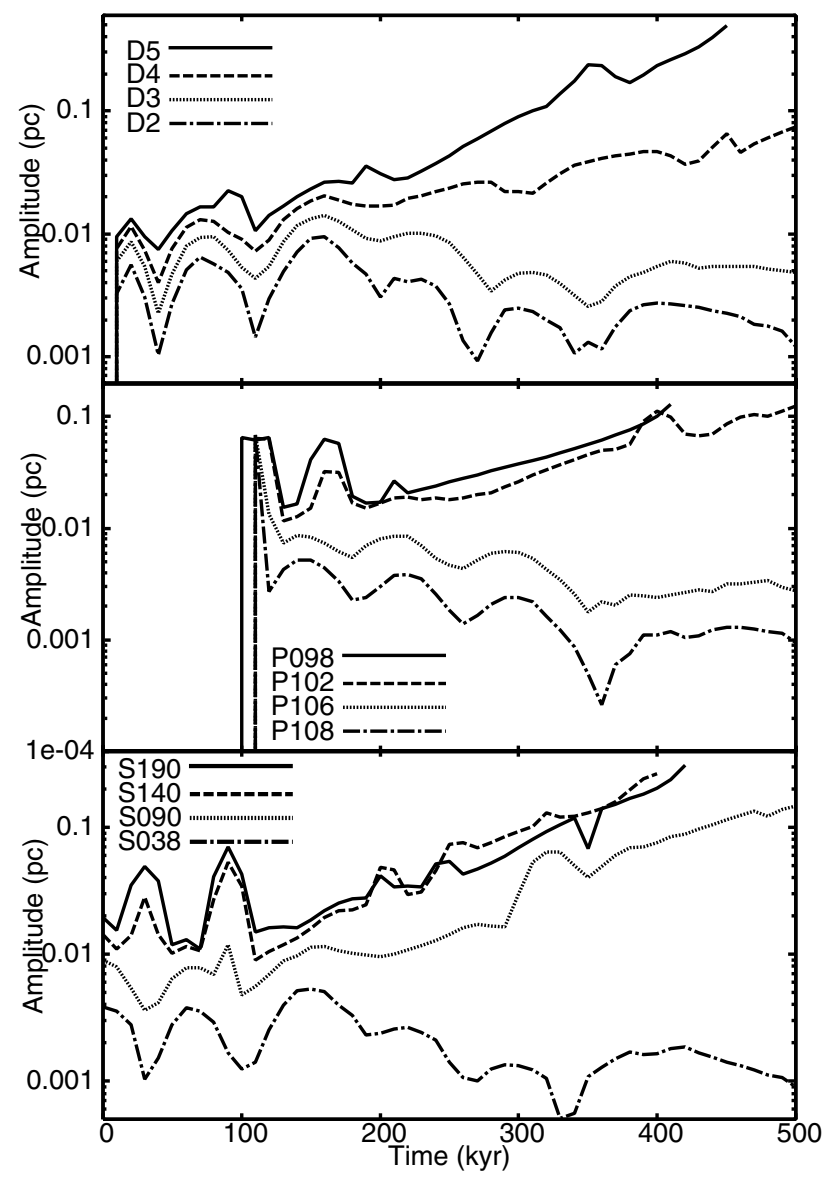

Fig. 4.- The time evolution of the amplitude for different methods of imposing the initial perturbation. Top: the initial perturbation is imposed in density. Middle: the perturbation is imposed in the photon number flux. Bottom: a surface perturbation is imposed. Note the amplitude is defined as half the peak-to-valley height of the perturbation in the $f=$ 0.5 contour in the $y$ direction. This does not always corresponds to the amplitude of the perturbation on the surface of the molecular cloud due to the "separation effect" (see text). When the imposed initial perturbation becomes large enough, the perturbations grow in all cases. When the initial perturbation is small, the growth is stabilized by recombination. 

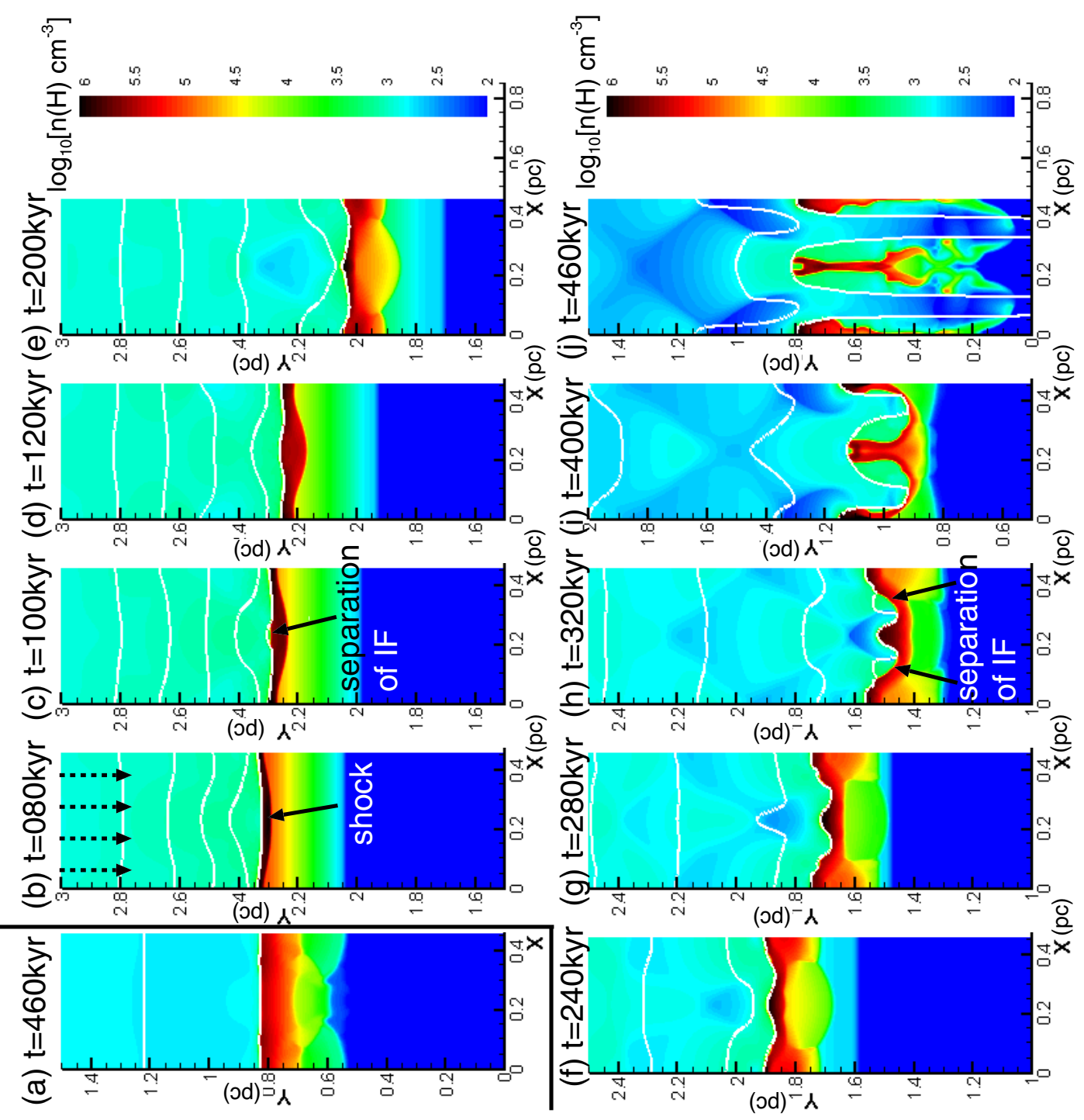

Fig. 5. - Log density (color) and incident photon flux contours (white solid curves) from 0 to $|\boldsymbol{J}|=5 \times 10^{11} \mathrm{~cm}^{-2} \mathrm{~s}^{-1}$ with uniform intervals of $|\boldsymbol{J}|=1 \times 10^{11} \mathrm{~cm}^{-2} \mathrm{~s}^{-1}$. The incident photon flux, which is parallel to the $y$ axis, comes in from the top boundary. The dashed arrows in (b) indicate the initial photon flux from the top boundary. (a) A 30\% initial perturbation in density is imposed (model D3), and no growth has occurred even at late times $(t=460 \mathrm{kyr})$. (b-j) Similar to (a) except than the initial density perturbation is $50 \%$ (model D5). The times corresponding to each 2D density snapshot are indicated at the top of each plot. (b) A shock is passing through the cloud, as indicated by the arrow. Note the $|\boldsymbol{J}|=1 \times 10^{11} \mathrm{~cm}^{-2} \mathrm{~s}^{-1}$ contour has the opposite phase as the IF. (c-g) The shock breaks out the back of the molecular cloud, and the acceleration phase begins. The solid arrows in (c) and (h) indicate the IF separation from the high density molecular cloud "surface". (h)-(i) Due to recombination, the IF separates from the high-density molecular cloud surface, which locally lowers the ablation pressure, and in (i) allows the perturbation spike in the center to grow due to the local pressure difference. (j) Once the perturbation growth exceeds a critical amplitude, the stabilization effect is reduced, and the evolution quickly enters the deep nonlinear regime, creating a pillar. 


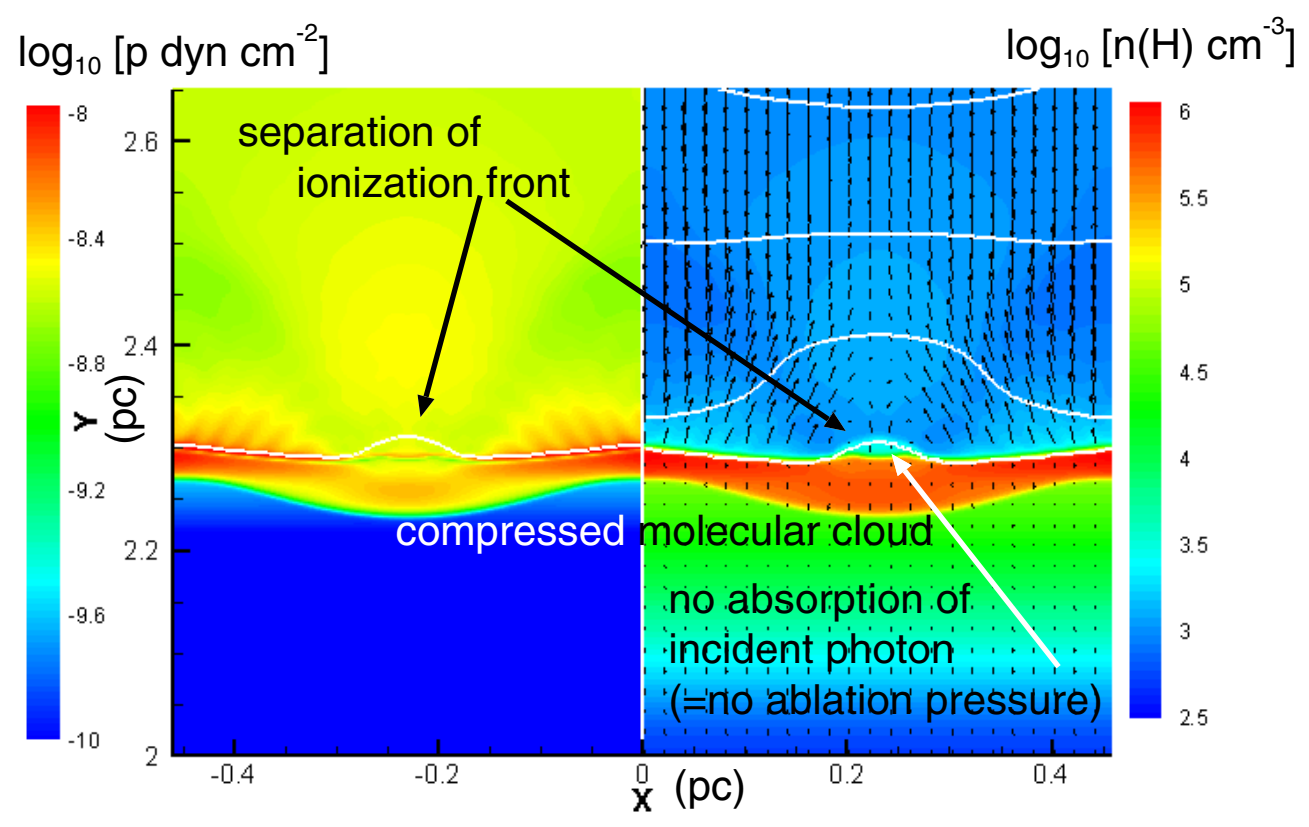

Fig. 6. - A close up of Fig. 5(c) around the IF. Density $(x>0)$ and pressure $(x<0)$ are plotted (color). The contours of the incident photon flux $\left(|\boldsymbol{J}|=0,1 \times 10^{11}, 2 \times 10^{11}, 3 \times\right.$ $\left.10^{11}, 4 \times 10^{11} \mathrm{~cm}^{-2} \mathrm{~s}^{-1}\right)$ are plotted $(x>0)$. The contour of the ionization degree $(f=0.5)$ is plotted $(x<0)$. The arrows in the right panel are velocity field. The IF slightly separated from the cloud surface at around $x=0.23, y=2.3 \mathrm{pc}$, since all the incident photons are absorbed by the recombined hydrogen in the high density region (around $x=0.23, y=2.4$ ). A slight expansion of the cloud in the $y$ direction can be seen. 

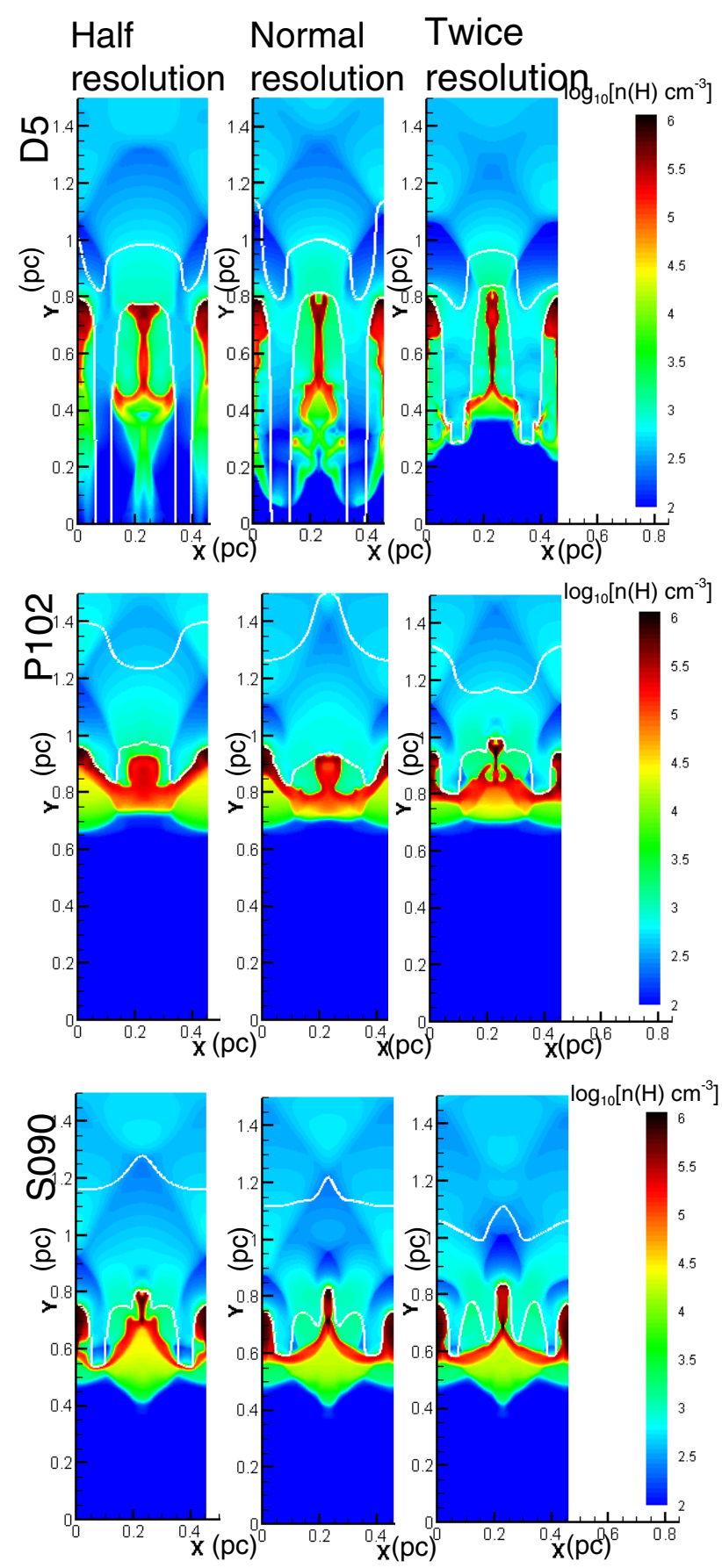

Fig. 7.- Lower (left panels: half the number of grid points in $x$ ), standard zoning (middle panels) and higher (right panels: twice the number of grid points in $x$ ) resolution calculations. The contours show the same physical values as in Fig.5. The top panels are model D5 at $t=460 \mathrm{kyr}$, the middle panels are model 102 at $t=440 \mathrm{kyr}$, and the bottom panels are S090 at $t=460 \mathrm{kyr}$. All the cases show large growth of the second harmonic of the imposed initial perturbation, differing only in the fine-scale structure. 


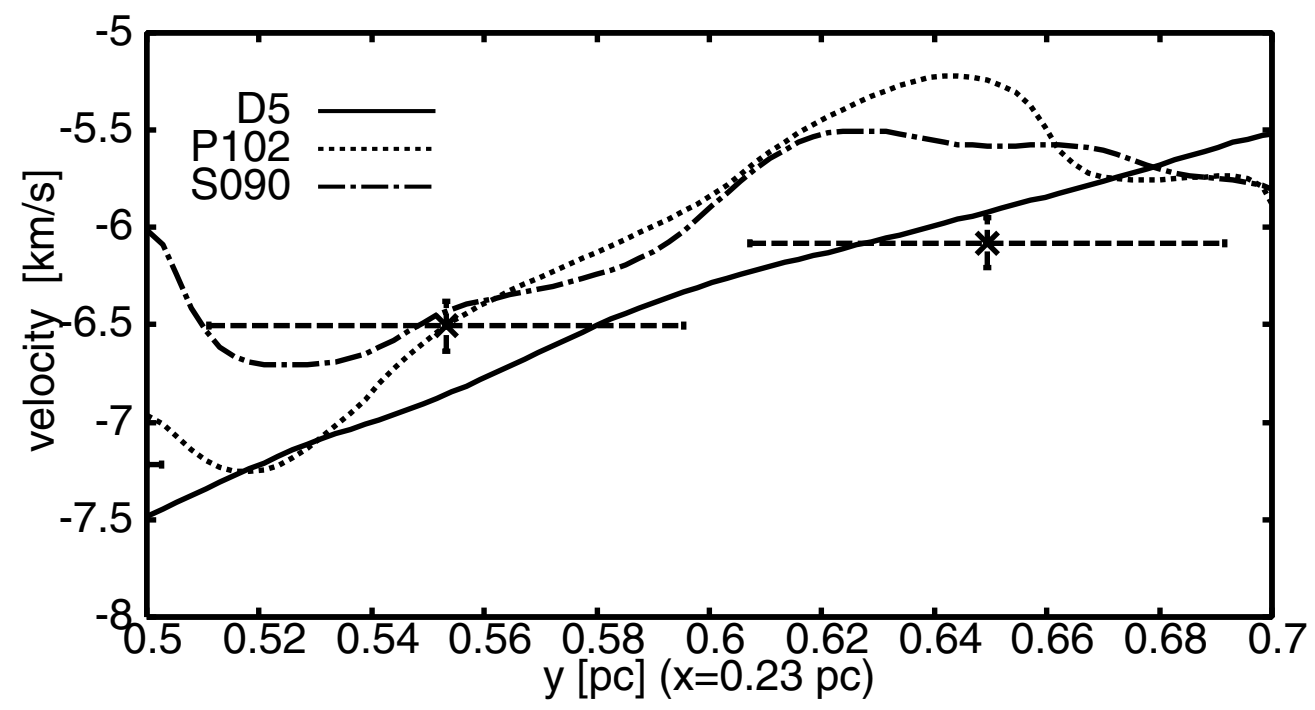

Fig. 8. - The velocity profile in the $y$ direction at $x=0.23 \mathrm{pc}$, for the different methods of initial perturbation: D5 $(t=460 \mathrm{kyr}), \mathrm{P} 102(t=480 \mathrm{kyr})$, and $\mathrm{S} 090(t=480 \mathrm{kyr})$. The pillar is oriented such that the right side represents its top and the left side represents its bottom. The points with error bars show the measured radial gas velocities in Pillar II of the Eagle Nebula from Pound (1998), divided by $\sin (15)$ to account for the line-ofsight inclination. The velocity gradients of models are in good agreement with the observed gradient. 